\title{
LOS OBJETOS NODRIZA COMO REFUGIO Y FUENTE DE NUTRIENTES: REFLEXIONES SOBRE EL ESTABLECIMIENTO Y RESTAURACIÓN DE CACTÁCEAS EN ZONAS ÁRIDAS DE LA VERTIENTE OCCIDENTAL DE LOS ANDES
}

\section{THE NURSE OBJECTS AS SHELTER AND NUTRIENTS SOURCE: REFLECTIONS ON CACTACEAE ESTABLISHMENT AND RESTORATION IN ARID ZONES BELONGING TO THE ANDEAN WESTERN SLOPES}

\author{
David A. Ramírez Collantes ${ }^{1}$
}

\begin{abstract}
Resumen
Se analizan dos recientes paradigmas referentes al reclutamiento y establecimiento de cactáceas. El primero de ellos está ligado al efecto facilitador que proporcionan los "objetos nodriza" (rocas) en comparación con el que brindan las "plantas nodriza", y que puede ser particularmente relevante en contextos donde la deposición de agua de nieblas y la formación de rocío son fenómenos de alta ocurrencia. El segundo paradigma tiene que ver con la biología trófica de cactáceas rupícolas. Trabajos recientes han descubierto y aislado bacterias que viven dentro (endofíticas) y fuera (en el rizoplano) de las raíces de los cactus, y que son responsables de la meteorización de las rocas y la posterior liberación de nutrientes. El estudio de la ganancia de agua "oculta" promovida por las rocas, así como, el aislamiento e inoculación de cepas de bacterias en plántulas de cactus prometen ser líneas de investigación de suma importancia en los planes de restauración y recuperación de cactáceas endémicas de las zonas áridas de la vertiente occidental de los andes.

Palabras clave: bacterias promotoras del crecimiento vegetal, cactáceas, objetos nodriza, niebla, plantas nodriza, meteorización de rocas, rocío.
\end{abstract}

\begin{abstract}
In this scientific note I analyze two new paradigms in the Cactaceae recruitment and establishment. The first one is related to the facilitation effect given by "nurse objects" (rocks) compared with "nurse plants", which could be enhanced in places where fog deposition and dew formation are highly frequent phenomena. The trophic biology of the rock-colonizing cactus is the second paradigm. Recent studies have discovered and isolated bacteria living into (endophytic) and outside (in the rhizoplane) cactus roots which are responsible for rock weathering and nutrients release. Research on the "hidden" water gains driven by rocks and bacteria isolation and inoculation to cactus seedlings are very important topics in the endemic cactus populations' restoration and recovering plans in arid environments of the Andean western slopes.

Key words: Cactaceae, dew, fog, nurse plants, nurse objects, plant growth promoting bacteria, rock weathering.
\end{abstract}

\section{Introducción.}

El conocimiento de los principales procesos que influyen sobre el funcionamiento y establecimiento de la vegetación es crucial para definir estrategias y llevar a cabo la restauración y/o revegetación de un determinado medio. Por ejemplo, en algunas zonas del Sahel, los suelos áridos e infértiles se caracterizan por la presencia de una costra física que reduce la infiltración de agua en el suelo luego de eventos de lluvia. La incorporación de "mulch" con termitas en dicho suelo ha permitido la recuperación de la infiltración promoviendo un incremento de la biomasa vegetal de 0 a 3,3 Tn/ha a los tres años desde el inicio de las actividades de manejo (de Goede \& Brussaard,
2002). Por otro lado, en zonas semiáridas australianas el acopio de troncos y ramas de árboles entre la vegetación sirvió como barrera de sedimentos que son acarreados por el agua que escurre tras la lluvia (Tongway et al., 2004). Dichos troncos y ramas por tanto simulan sumideros de recursos en el paisaje creando zonas de acumulación de nutrientes y de mayor infiltración propiciando a su vez la colonización de especies vegetales (Ludwig \& Tongway, 1996; Tongway \& Ludwig, 1996).

En zonas áridas con escasa ocurrencia de lluvias, los ingresos de agua que provienen de la deposición de niebla y la formación de rocío son de vital importancia para el funcionamiento de los ecosistemas 
(Henschel \& Seely, 2008). Así por ejemplo, algunos valores máximos de agua de niebla capturados por "neblinómetros" (Schemenauer et al., 1987) y reportados para desiertos de Namibia $\left(1 \mathrm{~mm} \mathrm{día}^{-1}\right.$; Henschel \& Seely, 2008), de la costa oeste de Sudáfrica (5,7 mm día ${ }^{-1}$; Olivier, 2002) y del Atacama (7 mm día ${ }^{-1}$; Cereceda et al., 2008) dan testimonio de la cantidad potencial de agua que estas zonas áridas pueden recibir por medio de estructuras que aumentan la superficie de interceptación de nieblas. Las rocas grandes distribuidas en las laderas cumplen un rol fundamental no solo en la captación de estas nieblas, sino que además pueden propiciar la presencia de rocío matutino ya que su enfriamiento durante la noche permitiría que la capa de aire circundante a su superficie alcance la temperatura de rocío (requisito fundamental para que se lleve a cabo la condensación de agua; Agam \& Berliner, 2006) en temporadas con ausencia de nieblas.

Los objetos nodriza frente a las plantas nodriza.

En zonas áridas de Méjico y EEUU, el establecimiento de cactáceas y numerosas especies de plantas suculentas ha sido relacionado con la presencia de arbustos, de tal manera que se ha propuesto una interacción de facilitación arbusto-cactus (Drezner \& Garrity, 2003; Drezner, 2006; Valiente-Banuet et al., 1991; Zúñiga et al., 2005). El arbusto actúa como "planta nodriza" que genera un micro-sitio óptimo para su "protegido" (cactus) propiciando: - sombreado y por lo tanto atenuación de altas radiaciones, materia orgánica y por lo tanto nutrientes derivados de la descomposición de hojarasca proveniente del arbusto, y - mayor disponibilidad de agua que es mantenida debajo de la planta nodriza. Sin embargo, en una reciente revisión de estudios realizados en diferentes zonas áridas y semiáridas del mundo, Maestre et al. (2005) encontraron que bajo condiciones de alto estrés ambiental las interacciones de competencia son más importantes que las de facilitación. La escasez de recursos limitantes (principalmente el agua) determina que las plantas nodriza compitan por éstos con las plantas protegidas, fenómeno que en algunas partes del mundo se ha denominado "sombra seca" (Valladares et al., 2004). Bajo este tipo de escenarios, los "objetos nodriza" como las rocas pueden desempeñar un rol facilitador similar al de los arbustos (a excepción de la deposición de hojarasca) permitiendo un ambiente con pocas variaciones de temperatura y humedad (Nobel et $a l ., 1992)$, evitando pérdidas de agua por evaporación (Reyes-Olivas et al., 2002) y con la ventaja de que no compiten con las plantas protegidas por los escasos recursos (Munguia-Rosas \& Sosa, 2008; Peters et al., 2008).

Los ambientes áridos donde habitan cactáceas poseen peculiaridades meteorológicas. Así, por ejemplo, mientras las cactáceas del desierto de Sonora en Méjico pueden recibir más de 174 mm/año de lluvias principalmente proveída por sistemas de convección tropicales asociadas a huracanes (Hastings et al., 2005), en el desierto de Atacama, considerado el más árido del mundo (Rundel et al., 1991), las cactáceas tienen que soportar promedios anuales extremos de sequía de hasta $0,8 \mathrm{~mm} /$ año (obtenido a partir de 30 años de registro meteorológico; Cereceda et al., 2008). Bajo este último escenario, es posible predecir que las rocas como "objetos nodriza" tengan un mayor peso que las "plantas nodriza" en la facilitación del reclutamiento de cactáceas. Esto último ha sido sugerido por algunos trabajos en cactáceas de la serranía de Lima - Perú. Castro et al. (2006) encontraron mayor supervivencia de plántulas de Melocactus peruvianus Vaupel y Haageocereus pseudomelanostele Subsp. aureispinus (Rauh \& Backeberg) Ostolaza en condiciones de sombra; teniendo en cuenta la escasa cobertura vegetal arbustiva en la zona, es de esperarse que dicho factor sea promovido por la presencia de rocas.

¿Los objetos nodriza como fuente de nutrientes?

Importantes densidades de cactáceas (principalmente individuos jóvenes) pertenecientes al género Pachycereus, Stenocereus y Mamillaria se encuentran en roquedales volcánicos en las zonas áridas de Baja California - Méjico (Bashan et al., 2002). Las raíces de esos individuos crecen adosadas a la roca sin suelo consolidado a su alrededor (medio denominado "aeropónico"), característica observada también en algunas cactáceas de la vertiente occidental de los andes peruanos (Figura 1). Microfotografías de la superficie de dichas raíces así como de su estructura interna, han revelado la presencia de bacterias en el rizoplano (Puente et al., 2004a) así como bacterias endofíticas (Puente et al., 2009a; Puente et al., 2009b), respectivamente. El aislamiento de dichas bacterias y su inoculación en rocas volcánicas pulverizadas ha demostrado que dichos organismos son capaces de: - degradar la roca en fragmentos más pequeños, - segregar compuestos orgánicos y protones (reduciendo el $\mathrm{pH}$ ), - fijar nitrógeno atmosférico y además - meteorizar la roca dejando disponible elementos como $\mathrm{P}, \mathrm{K}, \mathrm{Mg}, \mathrm{Mn}$, $\mathrm{Fe}, \mathrm{Cu}$ y Zn (Bashan et al., 2002; Puente et al., 2004a; Puente et al., 2004b; Puente et al., 2009a; Puente et al., 2009b;). Semillas del cactus Pachycereus pringlei ("cardón gigante") inoculadas con las mencionadas bacterias (de los géneros: Bacilus, Citrobacter, Klebsiella y Staphylococcus) y otras comerciales denominadas como "promotoras del crecimiento vegetal" (Azospirillum brasilense y Pseudomona putida) han generado plántulas del "cardón gigante" con mayor biomasa, elongación y volumen en sustrato formado únicamente por roca pulverizada (Puente et al., 2004b; Puente et al., 2009b). Sin embargo, no todas las rocas poseen el mismo potencial para la colonización y establecimiento de cactáceas. Estudios realizados en poblaciones de individuos jóvenes de 
Mammillaria fraileana al sur del desierto de Sonora, han demostrado que ésta cactácea es más dominante en tipo de rocas compuestas de minerales más susceptibles a la meteorización con dominancia de cristales volcánicos relacionados con riolitas, dacitas y andesitas, frente a sitios con rocas con más plagioclasas y piroxenos (Lopez et al., 2009).

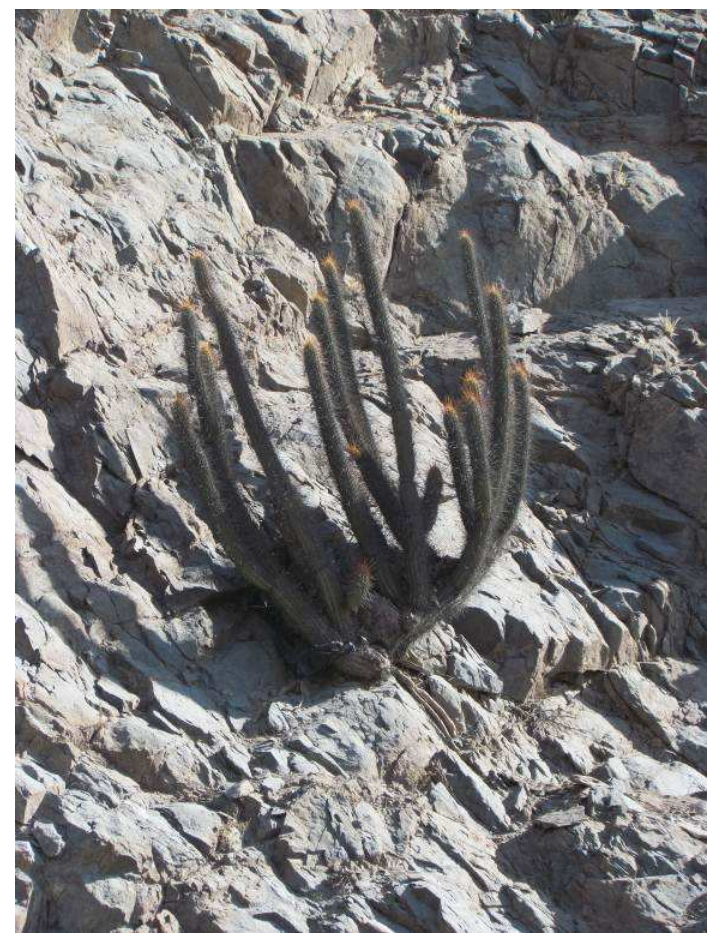

Figura 1. Individuo de Weberbauer weberbauerocereus

rocas, promueven el crecimiento de dichas plántulas en medios degradados (Bacilio et al., 2006; Bashan et al., 1999; Bashan et al., 2009). Estas premisas pueden abrir una línea de investigación que beneficiaría programas de restauración y recuperación de cactáceas endémicas. En tal esfuerzo se requerirá un trabajo conjunto entre ecólogos, microbiólogos e hidrólogos.

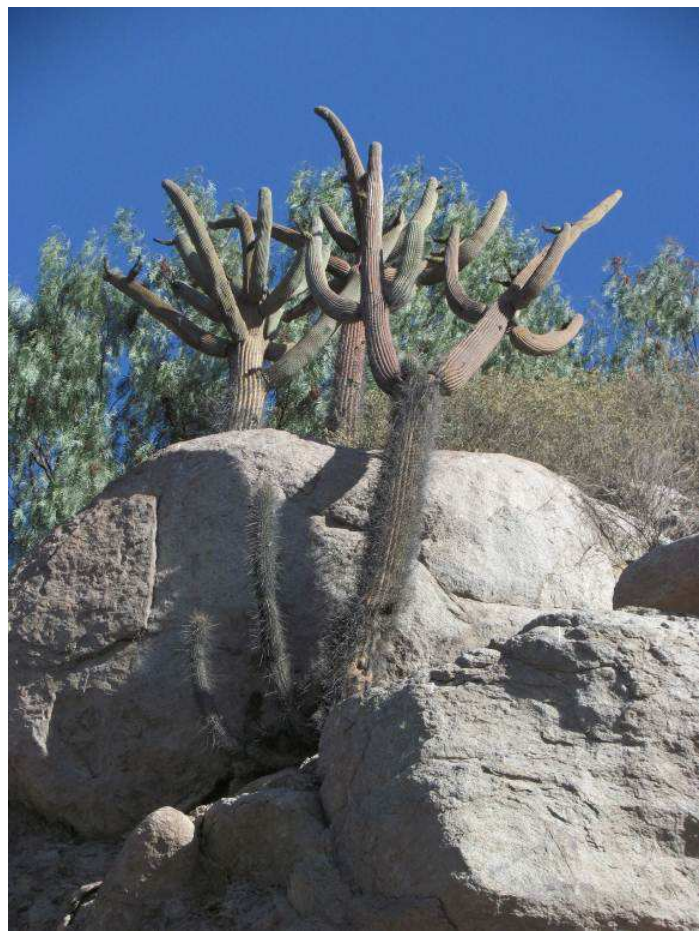

(K. Schum. Ex Vaupel) Backeb (izquierda) y Browningia candelaris (Meyen) Britton \& Rose (derecha) creciendo sobre rocas en la serranía esteparia del sur de los Andes peruanos (Localidad de "Virgen de las Peñas", distrito de Uchumayo, Departamento de Arequipa, Perú) a 2500 m.s.n.m. Los individuos de la izquierda y derecha miden aproximadamente 2,5 y 3,5 $\mathrm{m}$ de altura máxima respectivamente. Fotografías tomadas por el autor.

\section{Conclusión.}

Más estudios son necesarios para cuantificar el efecto causal de la facilitación de las rocas en zonas de la vertiente occidental de los Andes. En estos medios, además de los efectos positivos que pueden tener las rocas como "objeto nodriza", se suma el ingreso de agua por nieblas y rocío. La medición de estos ingresos de agua "oculta" podría abordarse mediante el empleo de mini-lisímetros y sensores en continuo de humedad del suelo aledaño a rocas (ver por ejemplo Ramírez et al., 2007; Villagarcia et al., 2007). Tales evaluaciones también podrían contribuir a discernir el tamaño óptimo de roca, así como la orientación y la altitud de ladera que promuevan un mayor ingreso de agua. Por otro lado, la experiencia en restauración de plántulas de cactáceas columnares en medios oligotróficos ha demostrado que el uso de enmiendas orgánicas (compost) a bajas concentraciones combinado con suelo oriundo, y la inoculación de semillas con bacterias "promotoras de crecimiento vegetal" y cepas capaces de meteorizar

\section{Referencias bibliográficas.}

Agam N. \& Berliner P.R. (2006). Dew formation and water vapor adsorption in semi-arid environments - A review. Journal of Arid Environments 65:572-590.

Bacilio M., Hernandez J.P. \& Bashan Y. (2006). Restoration of giant cardon cacti in barren desert soil amended with common compost and inoculated with Azospirillum brasilense. Biology and Fertility of Soils 43: 112-119.

Bashan Y., Rojas A. \& Puente M.E. (1999). Improved establishment and development of three cactus species inoculated with Azospirillum brasilense transplanted into disturbed urban desert soil. Canadian Journal of Microbiology 45:441-451.

Bashan Y., Li C.Y., Lebsky V.K., Moreno M. \& de-Bashan L.E. (2002). Primary colonization of volcanic rocks by plants in arid Baja California, Mexico. Plant Biology 4: 392-402.

Bashan Y., Salazar B., Puente M.E., Bacilio M. \& Linderman R. (2009). Enhanced establishment and growth of giant cardon cactus in an eroded field in the Sonoran Desert using native legume trees as nurse plants aided by plant growth-promoting microorganisms and compost. Biology and Fertility of Soils 45: 585-594. 
Castro V., Eyzaguirre R. \& Ceroni A. (2006). Supervivencia de plántulas de Melocactus peruvianus Vaupel y Haageocereus pseudomelanostele subsp. aureispinus (Rauh \& Backeberg) Ostolaza, en condiciones experimentales. Cerro Umarcata, Valle del Río Chillón, Lima. Ecología Aplicada 5 (1,2): 61-66.

Cereceda P., Larrain H., Osses P., Farias M. \& Egana I. (2008). The climate of the coast and fog zone in the Tarapaca Region, Atacama Desert, Chile. Atmospheric Research 87:301-311.

de Goede, R.G.M. \& Brussaard, L. (2002). Soil zoology: an indispensable componente of integrated ecosystem studies. European Journal of Soil Biology 38:1-6.

Drezner T.D. (2006). Plant facilitation in extreme environments: the non-random distribution of saguaro cacti (Carnegiea gigantea) under their nurse associates and the relationship to nurse architecture. Journal of Arid Environments 65, 46-61.

Drezner T.D. \& Garrity C.M. (2003). Saguaro distribution under nurse plants in Arizona's Sonoran Desert: directional and microclimate influences. Professional Geographer 55, 505-512.

Hastings S.J., Oechel W.C. \& Muhlia-Melo A. (2005). Diurnal, seasonal and annual variation in the net ecosystem $\mathrm{CO} 2$ exchange of a desert shrub community (Sarcocaulescent) in Baja California, Mexico. Global Change Biology 11: 927-939.

Henschel J.R. \& Seely M.K. (2008). Ecophysiology of atmospheric moisture in the Namib Desert. Atmospheric Research 87: 362-368.

Lopez B.R., Bashan Y., Bacilio M. \& De la Cruz-Aguero G. (2009). Rock-colonizing plants: abundance of the endemic cactus Mammillaria fraileana related to rock type in the southern Sonoran Desert. Plant Ecology 201: 575-588.

Ludwig J.A. \& Tongway D.J. (1996). Rehabilitation of semi-arid landscapes in Australia II: restoring productive soil patches. Restoration Ecology 4: 398-406.

Maestre F.T., Valladares F. \& Reynolds J.F. (2005). Is the change of plant-plant interactions with abiotic stress predictable? A meta-analysis of field results in arid environments. Journal of Ecology 93: 748-757.

Munguia-Rosas M.A. \& Sosa V.J. (2008). Nurse plants vs. Nurse objects: Effects of woody plants and rocky cavities on the recruitment of the Pilosocereus leucocephalus columnar cactus. Annals of Botany 101: 175-185.

Nobel P.S., Miller P.M. \& Graham E.A. (1992). Influence of Rocks on Soil-Temperature, Soil-Water Potential, and Rooting Patterns for Desert Succulents. Oecologia 92: 90-96.

Olivier J. (2002). Fog-water harvesting along the West Coast of South Africa: a feasibility study. Water SA 28:349-360

Peters E.M., Martorell C. \& Ezcurra E. (2008). Nurse rocks are more important than nurse plants in determining the distribution and establishment of globose cacti (Mammillaria) in the Tehuacan Valley, Mexico. Journal of Arid Environments 72:593-601.
Puente M.E., Bashan Y., Li C.Y. \& Lebsky V.K. (2004a). Microbial populations and activities in the rhizoplane of rock-weathering desert plants. I. Root colonization and weathering of igneous rocks. Plant Biology 6: 629-642.

Puente M.E., Li C.Y. \& Bashan Y. (2004b). Microbial populations and activities in the rhizoplane of rockweathering desert plants. II. Growth promotion of cactus seedlings. Plant Biology 6: 643-649.

Puente M.E., Li C.Y. \& Bashan Y. (2009a). Rock-degrading endophytic bacteria in cacti. Environmental and Experimental Botany 66: 389-401.

Puente M.E., Li C.Y. \& Bashan Y. (2009b). Endophytic bacteria in cacti seeds can improve the development of cactus seedlings. Environmental and Experimental Botany 66: 402-408.

Ramírez D.A., Bellot J., Domingo F. \& Blasco A. (2007). Can water responses in Stipa tenacissima L. during the summer season be promoted by non-rainfall water gains in soil? Plant and Soil 291: 67-79.

Reyes-Olivas A., Garcia-Moya E. \& Lopez-Mata L. (2002). Cacti-shrub interactions in the coastal desert of northern Sinaloa, Mexico. Journal of Arid Environments 52: 431445.

Rundel, P.W., Dillon, M.O., Palma, B., Mooney, H.A., Gulmon, S.L. \& Ehleringer, J.R. (1991). The phytogeography and ecology of the coastal Atacama and Peruvian deserts. Aliso 13(1): 1-50.

Schemenauer, R.S., Cereceda, P. \& Carvajal, N. (1987). Measurements of Fog Water Deposition and Their Relationships to Terrain Features. Journal of Climate and Applied Meteorology 26: 1285-1291.

Tongway D.J. \& Ludwig J.A. (1996) Rehabilitation of semi-arid landscapes in Australia II: restoring productive soil patches. Restoration Ecology 4: 388-397.

Tongway D. J., Cortina J. \& Maestre F.T. (2004) La gestión de la heterogeneidad espacial en medios semiáridos. Ecosistemas $2004 / 1$ (url:www.aeet.org/ecosistemas/041/revision5.htm).

Valiente-Banuet A., Bolongaro-Crevena A., Briones O., Ezcurra E., Rosas M., Núñez H., Barnard G. \& Vázquez E. (1991). Spatial relationships between cacti and nurse shrubs in a semi-arid environment in central Mexico. Journal of Vegetation Science 2, 15-20.

Valladares F., Aranda I. \& Sánchez-Gómez D. (2004). La luz como factor ecológico y evolutivo para las plantas y su interacción con el agua, En: F. Valladares (Ed.), Ecología del bosque mediterráneo en un mundo cambiante, Ministerio del Medio Ambiente, EGRAF, S.A., Madrid. pp. 335-369.

Villagarcia L., Were A., Domingo F., Garcia M. \& AladosArbotedas L. (2007). Estimation of soil boundary-layer resistance in sparse semiarid stands for evapotranspiration modelling. Journal of Hydrology 342: 173-183.

Zúñiga B., Malda G. \& Suzan H. (2005). Interacciones Planta-Nodriza en Lophophora diffusa (Cactaceae) en un Desierto Subtropical de México. Biotropica 37, 351356.

\footnotetext{
${ }^{1}$ http://davidramirezc.weebly.com. Profesor Visitante, Departamento Académico de Biología, Universidad Nacional Agraria La Molina, Lima - Perú E-mail: dramirez@lamolina.edu.pe.
} 